\title{
Interstitial Fibrosis In Heart Failure
}


eBook ISBN: $\quad 0-387-22825-X$

Print ISBN: $\quad$ 0-387-22824-1

(C2005 Springer Science + Business Media, Inc.

Print (C2005 Springer Science + Business Media, Inc.

Boston

All rights reserved

No part of this eBook may be reproduced or transmitted in any form or by any means, electronic, mechanical, recording, or otherwise, without written consent from the Publisher

Created in the United States of America

Visit Springer's eBookstore at: and the Springer Global Website Online at: http://www.ebooks. kluweronline.com

http://www.springeronline.com 


\title{
Interstitial Fibrosis In Heart Failure
}

\author{
edited by
}

Francisco J. Villarreal, M.D., Ph.D.

Professor

Department of Medicine

University of California, San Diego

School of Medicine

San Diego, California

Springer 


\section{Developments in Cardiovascular Medicine}

232. A. Bayés de Luna, F. Furlanello, B.J. Maron and D.P. Zipes (eds.): Arrhythmias and Sudden Death in Athletes. $2000 \quad$ ISBN: 0-7923-6337-X

233. J-C. Tardif and M.G. Bourassa (eds): Antioxidants and Cardiovascular Disease. 2000.

ISBN: 0-7923-7829-6

234. J. Candell-Riera, J. Castell-Conesa, S. Aguadé Bruiz (eds): Myocardium at Risk and Viable Myocardium Evaluation by SPET. 2000.ISBN: 0-7923-6724-3

235. M.H. Ellestad and E. Amsterdam (eds): Exercise Testing: New Concepts for the New Century. 2001.

ISBN: 0-7923-7378-2

236. Douglas L. Mann (ed.): The Role of Inflammatory Mediators in the Failing Heart. 2001 ISBN: 0-7923-7381-2

237. Donald M. Bers (ed.): Excitation-Contraction Coupling and Cardiac Contractile Force, Second Edition. 2001

ISBN: 0-7923-7157-7

238. Brian D. Hoit, Richard A. Walsh (eds.): Cardiovascular Physiology in the Genetically Engineered Mouse, Second Edition. $2001 \quad$ ISBN 0-7923-7536-X

239. Pieter A. Doevendans, A.A.M. Wilde (eds.): Cardiovascular Genetics for Clinicians 2001

ISBN 1-4020-0097-9

240. Stephen M. Factor, Maria A.Lamberti-Abadi, Jacobo Abadi (eds.): Handbook of Pathology and Pathophysiology of Cardiovascular Disease, 2001

ISBN $\quad 0-7923-7542-4$

241. Liong Bing Liem, Eugene Downar (eds): Progress in Catheter Ablation. 2001 ISBN $1-4020-0147-9$

242. Pieter A. Doevendans, Stefan Kääb (eds): Cardiovascular Genomics: New Pathophysiological Concepts. 2002 ISBN 1-4020-7022-5

243. Daan Kromhout, Alessandro Menotti, Henry Blackburn (eds.): Prevention of Coronary Heart Disease: Diet, Lifestyle and Risk Factors in the Seven Countries Study. 2002 ISBN 1-4020-7123-X

244. Antonio Pacifico (ed.), Philip D. Henry, Gust H. Bardy, Martin Borggrefe, Francis E. Marchlinski, Andrea Natale, Bruce L. Wilkoff (assoc. eds): Implantable Defibrillator Therapy: A Clinical Guide, 2002 ISBN 1-4020-7143-4

245. Hein J.J. Wellens, Anton P.M. Gorgels, Pieter A. Doevendans (eds.): The ECG in Acute Myocardial Infarction and Unstable Angina: Diagnosis and Risk Stratification. 2002 ISBN 1-4020-7214-7

246. Jack Rychik, Gil Wernovsky (eds.): Hypoplastic Left Heart Syndrome. 2003 ISBN 1-4020-7319-4

247. Thomas H. Marwick: Stress Echocardiography. Its Role in the Diagnosis and Evaluation of Coronary Artery Disease 2nd Edition. ISBN 1-4020-7369-0

248. Akira Matsumori: Cardiomyopathies and Heart Failure: Biomolecular, Infectious and Immune Mechanisms. 2003

249. Ralph Shabetai: The Pericardium. 2003 ISBN 1-4020-7438-7

250. Irene D. Turpie; George A. Heckman (eds.): Aging Issues in Cardiology. 2004 ISBN 1-40207674-6

251. C.H. Peels; L.H.B. Baur (eds.): Valve Surgery at the Turn of the Millennium. 2004 ISBN 1-4020-7834-X

252. Jason X.-J. Yuan (ed.): Hypoxic Pulmonary Vasoconstriction: Cellular and Molecular Mechanisms, 2004 ISBN 1-4020-7857-9

253. Francisco J. Villarreal (ed.): Interstitial Fibrosis In Heart Failure 2004

Previous volumes are still available

ISBN 0-387-22824-1 
TABLE OF CONTENTS

List of Contributors

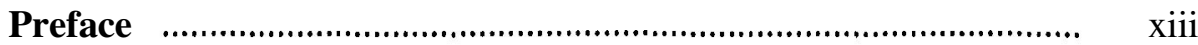

Acknowledgments

I. ARCHITECTURE OF THE NORMAL AND REMODELED HEART

1. The Architecture of the Heart: Myocyte Organization and the Cardiac Extracellular Matrix

Ian LeGrice, Adèle Pope and Bruce Smaill

2. Extracellular Matrix and Cardiac Remodeling

Bodh I. Jugdutt

3. Pro-Inflammatory Cytokines and Cardiac Extracellular Matrix: Regulation of the Fibroblast Phenotype

R. Dale Brown, M. Darren Mitchell and Carlin S. Long

4. Pharmacology of G-Protein-linked Signaling in Cardiac Fibroblasts

Sara A. Epperson, Asa B. Gustafsson, Annete M. Gonzalez,

Sonia Villegas, J. Gary Meszaros and Laurence L. Brunton

II. DIAGNOSTIC TOOLS FOR THE IDENTIFICATION OF MYOCARDIAL FIBROSIS AND CHANGES IN VENTRICULAR MECHANICS

5. Serum Markers of Fibrillar Collagen Metabolism in Cardiac Diseases

Javier Diez

6. Ultrasonic Characterization of the Myocardium

Mark R. Holland and Samuel A. Wickline

7. The Mechanics of the Fibrosed/Remodeled Heart

Robert C. Gorman, Benjamin, M. Jackson,

Joseph H. Gorman and L. Henry Edmunds Jr. 
8. Renin Angiotensin Aldosterone System and Cardiac Extracellular Matrix

Yao Sun and Karl T. Weber …............................................. 167

9. Matrix Metalloproteinases and Myocardial Remodeling

English Chapman and Francis G. Spinale

10. Cardiac Mast Cells as Mediators of Ventricular

Remodeling

Joseph S. Janicki, Gregory L. Brower, Amanda L. Chancey,

Mary F. Forman and Lynetta J. Jobe

IV. MATRIX METALLOPROTEINASES IN CARDIAC REMODELING

11. Turmoil in the Cardiac Myocyte: Acute Intracellular Activation of Matrix Metalloproteinases

Manoj M. Lalu, Hernando Leon, Richard Schulz

12. Regulation of Cardiac Extracellular Matrix

Remodeling Following Myocardial Infarction

Jack P. M. Cleutjens

13. Experimental Models of MMP Activation: Ventricular Volume Overload

Baljit S. Walia, Stephen C. Jones, Jianming Hao

Ian M.C. Dixon 
14. Targets for Pharmacological Modulation of Cardiac Fibrosis

Lindsay Brown, Vincent Chan and Andrew Fenning

15. Biological and Functional Effects of Chronic Mechanical Support Induced by Left Ventricular Assist Devices on Failing Human Myocardium

Guillermo Torre-Amione, Cynthia K. Wallace and O.H. Frazier

16. Molecular Strategies for the Prevention of Cardiac Fibrosis

Ramareddy V. Guntaka and Karl T. Weber

17. Prospects for Gene Therapy for the Fibrosed Heart: Targeting Regulators of Extracellular Matrix Turnover Hiroshi Ashikaga and Francisco Villarreal

18. Therapeutic Potential of TIMPs in Heart Failure

Suresh C. Tyagi

Index 


\section{List of Contributors}

Guillermo Torre-Amione, Texas Heart Institute at St. Luke's Episcopal Hospital, Houston, Texas, U.S.A.

Hiroshi Ashikaga, Department of Medicine, University of California San Diego, San Diego, CA, U.S.A.

Gregory L. Brower, Department of Anatomy, Physiology \& Pharmacology, Auburn University, Auburn, Alabama, U.S.A.

Dale Brown, Division of Cardiology, Denver Health Medical Center and University of Colorado Health Sciences Center, Denver, CO

Lindsay Brown, Department of Physiology and Pharmacology, School of Biomedical Sciences, The University of Queensland, Australia

Laurence L. Brunton, Department of Pharmacology, University of California San Diego, San Diego, CA, U.S.A.

Vincent Chan, Department of Physiology and Pharmacology, School of Biomedical Sciences, The University of Queensland, Australia

Amanda L. Chancey, Department of Anatomy, Physiology \& Pharmacology, Auburn University, Auburn, Alabama, U.S.A.

R. English Chapman, Medical University of South Carolina, Charleston, South Carolina, U.S.A.

Jack P. M. Cleutjens, Cardiovascular Research Institute Maastricht, Dept. Of Pathology, University Maastricht, Maastricht, The Netherlands

Javier Diez, Division of Cardiovascular Pathophysiology, School of Medicine, and Department of Cardiology and Cardiovascular Surgery, University Clinic, University of Navarra, Pamplona, Spain

Ian Dixon, Institute of Cardiovascular Sciences, St. Boniface General Hospital Research Centre, University of Manitoba, Winnipeg, Canada

L. Henry Edmunds, University of Pennsylvania, Philadelphia, PA, U.S.A. 
Sara Epperson, Department of Pharmacology, University of California San Diego, San Diego, CA, U.S.A.

Mary F. Forman, Department of Anatomy, Physiology \& Pharmacology, Auburn University, Auburn, Alabama, U.S.A.

Andrew Fenning, Department of Physiology and Pharmacology, School of Biomedical Sciences, The University of Queensland, Australia

O.H. Frazier, Texas Heart Institute at St. Luke's Episcopal Hospital, Houston, Texas, U.S.A.

Annette M. Gonzalez, Department of Pharmacology, University of California San Diego, San Diego, CA, U.S.A.

Asa Gustafsson, Department of Pharmacology, University of California San Diego, San Diego, CA, U.S.A.

Joseph H. Gorman, University of Pennsylvania, Philadelphia, PA, U.S.A.

Robert C. Gorman, University of Pennsylvania, Philadelphia, PA, U.S.A.

Ramareddy V. Guntaka, Departments of Molecular Sciences and Medicine, University of Tennessee Health Science Center, Memphis, Tennessee, U.S.A.

Jianming Hao, Institute of Cardiovascular Sciences, St. Boniface General Hospital Research Centre, University of Manitoba, Winnipeg, Canada

Mark R. Holland, Washington University in St. Louis, in St. Louis, MO, U.S.A.

Benjamin M. Jackson, University of Pennsylvania, Philadelphia, PA, U.S.A.

Lynetta J. Jobe, Department of Anatomy, Physiology \& Pharmacology, Auburn University, Auburn, Alabama, U.S.A.

Joseph S. Janicki, Department of Anatomy, Physiology \& Pharmacology, Auburn University, Auburn, Alabama, U.S.A.

Stephen C. Jones, Institute of Cardiovascular Sciences, St. Boniface General Hospital Research Centre, University of Manitoba, Winnipeg, Canada 
Bodh I. Jugdutt, Cardiology Division of the Department of Medicine, University of Alberta, Edmonton, Alberta, Canada

Manoj M. Lalu Cardiovascular Research Group, University of Alberta, Edmonton, Alberta, Canada

Ian Legrice, The Bioengineering Institute \& Department of Physiology, University of Auckland, Auckland, New Zealand

Hernando Leon, Cardiovascular Research Group, University of Alberta, Edmonton, Alberta, Canada

Carlin S. Long, Division of Cardiology, Denver Health Medical Center and University of Colorado Health Sciences Center, Denver, CO

J. Gary Meszaros, Department of Pharmacology, University of California San Diego, San Diego, CA, U.S.A.

M. Darren Mitchell, Division of Cardiology, Denver Health Medical Center and University of Colorado Health Sciences Center, Denver, CO U.S.A.

Adele Pope, The Bioengineering Institute \& Department of Physiology, University of Auckland, Auckland, New Zealand

Richard Schulz, Cardiovascular Research Group, University of Alberta, Edmonton, Alberta, Canada

Francis G. Spinale, Medical University of South Carolina, Charleston, South Carolina, U.S.A.

Bruce Smaill, The Bioengineering Institute \& Department of Physiology, University of Auckland, Auckland, New Zealand

Yao Sun, Division of Cardiovascular Diseases, Department of Medicine, University of Tennessee Health Science Center, Memphis, Tennessee, U.S.A.

Suresh C. Tyagi, Department of Physiology and Biophysics, University of Louisville School of Medicine, Louisville, Kentucky, U.S.A.

Francisco J. Villarreal, Department of Medicine, University of California San Diego, San Diego, CA, U.S.A. 
xii

Sonia Villegas, Department of Pharmacology, University of California San Diego, San Diego, CA, U.S.A.

Baljit S. Walia, Institute of Cardiovascular Sciences, St. Boniface General Hospital Research Centre, University of Manitoba, Winnipeg, Canada

Cynthia K. Wallace, Texas Heart Institute at St. Luke's Episcopal Hospital, Houston, Texas, U.S.A.

Karl T. Weber, Division of Cardiovascular Diseases, Department of Medicine, University of Tennessee Health Science Center, Memphis, Tennessee, U.S.A.

Samuel A. Wickline, Washington University in St. Louis, in St. Louis, MO, U.S.A. 


\section{Preface}

The presence of cardiac extracellular matrix (ECM) proteins within the myocardium allows the heart to fulfill critical physiological functions. However, the excessive deposition of ECM proteins (mostly collagens) within the myocardium can contribute to the transformation of the heart whose function relies on optimal mechanics into a dysfunctional organ. The increasing prevalence of heart failure in the general population has led to greater recognition of the importance that the excess deposition of ECM in the heart has in the pathophysiology of the disease. Most cardiologists would agree that in the presence of diffuse fibrosis a true reversal of adverse cardiac remodeling may not occur if the excess deposition of ECM proteins is not either significantly reduced or eventually eliminated. The cardiac fibroblast, which is the cell that produces most of the ECM in the heart, was essentially an ignored biological entity up until the early 1990's. Since then, the number of publications related to the cellular physiology of these cells has increase substantially. This fact highlights the importance given by basic scientists to the regulation of ECM production/deposition in the heart. Over the last decade or so, greater recognition has also been given the issue of ECM "turnover". Indeed, the excess deposition of ECM proteins in the myocardium not only reflects the greater production of the proteins but also their reduced degradation. In this regard, a significant number of publications have also emerged substantiating a role for matrix metalloproteinases in regulating the turnover of ECM proteins.

The major objective of this book is to provide a timely and integrative review of the basics of cardiac ECM structure, how the process of cardiac remodeling influences its disposition, abundance and function, possible noninvasive techniques for diagnosis, as well as discuss potential drug-based or molecular therapeutic strategies that may interrupt or even reverse the course of the development of cardiac fibrosis. The book is divided into 5 sections: I. Macro and microscopic architecture of the normal and remodeled heart; II. Myocardial fibrosis: Diagnosis and alterations in ventricular mechanics; III. Pathophysiology of cardiac remodeling and fibrosis; IV. Matrix metalloproteinases in cardiac remodeling and fibrosis; V. Therapeutic prospects for myocardial fibrosis. Chapter 1 highlights the architecture of the heart with a focus on the disposition of the ECM. Chapter 2 incorporates basic molecular, structural and biochemical features of the cardiac ECM into the context of pathological cardiac remodeling. Chapter 3 highlights features of the cardiac fibroblast and how this cell responds to known pro-inflammatory mediators. Chapters 4 and 5 focus on the possibility of utilizing non-invasive methodologies to detect either an ensuing fibrotic state or the presence of fibrosis. Chapter 6 then provides an overview on how the development of adverse ventricular remodeling and fibrosis impacts the mechanical behavior of 
the heart.

Chapters included in section III of the book focus on systems known to be important in the pathophysiology of cardiac remodeling/fibrosis including the roles played by the renin-angiotensin system (Chapter 7), matrix metalloproteinases (Chapter 8) and mast cells (Chapter 9). The following section (IV) then takes a more focused approach in describing the role that matrix metalloproteinases play in the pathophysiology of cardiac remodeling/fibrosis in the setting of ischemia-reperfusion injury (Chapter 10), myocardial infarction (Chapter 11), and volume overload (Chapter 12). The last section (V) covers the topic of potential therapeutic approaches for cardiac fibrosis. Chapter 13 comprehensively covers aspects related to pharmacotherapy including those currently used in the pre-clinical and clinical settings. The use of ventricular assist devices has become an attractive therapeutic option for those heart failure patients who are deemed suitable candidates. This topic is covered in Chapter 14. The observation that in some patients certain degrees of "reverse" remodeling can be achieved by the use of these assist devices has also focused attention on how ECM-related changes may follow this "temporary" treatment option. The implantation and subsequent removal of assist devices provides scientists with a unique opportunity, that of sampling diseased and "treated" heart tissue. It is interesting to note that in the limited number of reports published so far many of largest changes in cardiac gene expression levels prior to and after assist device treatment are in ECM-related genes. The last 2 chapters (15 and 16) cover the topic of prospects for molecular-based targeted therapy or gene therapy of the fibrosed heart.

In summary this book attempts to cover current state of the art findings relevant to cellular and molecular processes underlying cardiac fibrosis including basic elements of structure, function, diagnosis, and treatment. My hope is that this book can be of use to both scientists and clinicians. As heart failure continues to emerge as a major threat a greater focused effort will be required to attempt to mitigate or reverse its consequences.

\section{Acknowledgments}

This book is dedicated to my mentors Drs. James Covell, Wolfgang Dillmann and Laurence Brunton for continuously supporting me in the pursuit of my academic career. I would also like to take the opportunity to thank all contributors for their excellent chapters. Finally, I would also like to express my greatest appreciation for the preparation and editorial help provided by Ms. Maria L.Pacheco and Shirley Reynolds. 
I. ARCHITECTURE OF THE NORMAL AND REMODELED HEART 


\title{
Chapter 1
}

\section{The Architecture of the Heart: Myocyte Organization and the Cardiac Extracellular Matrix}

\author{
Ian LeGrice, Adèle Pope, Bruce Smaill \\ University of Auckland, Auckland, New Zealand
}

\section{Introduction}

Bioengineering research is providing new insights into the mechanical function of the heart and the extent to which this is underpinned by myocardial architecture and the cardiac extracellular matrix [1]. The normal left ventricle ejects more than $60 \%$ of its end-diastolic cavity volume during systole and analyses of left ventricular wall motion and regional deformation using imaging modalities such as MRI, reveal that this is due to relatively uniform shortening of myocytes across the ventricular wall, slippage or shearing of adjacent groups of myocytes, and the twisting or torsion of the ventricle about its axis.

Computer modeling studies indicate that this optimal mechanical performance is critically dependent on the geometry of the cardiac chambers and the organization of cardiac myocytes [1]. Cardiac myocytes and coronary vessels are embedded in a complex extracellular matrix that consists of collagen, elastin, fibronectin, laminin and proteoglycans [2]. The cardiac extracellular matrix is highly responsive to mechanical loading and plays a central role in the remodeling that occurs in response to altered loading conditions. To understand the functions of the extracellular connective tissue matrix more completely, we need to see them within a broader structural context. In this chapter, we will review the current understanding of myocardial architecture in the hope that it will provide a framework for integrating other more detailed information on the cardiac extracellular matrix found elsewhere in this monograph.

\section{The Organization of Myocytes in the Heart}

The cardiac muscle cell or myocyte is the main structural component of myocardium, occupying around $70 \%$ of ventricular wall volume under normal circumstances. Cardiac myocytes resemble ellipsoid cylinders with a major-axis 
dimension of 10 to $20 \mu \mathrm{m}$ and a length of 80 to $100 \mu \mathrm{m}$. Myocytes insert end to end and each is connected with several others to form a three-dimensional (3D) network of cells.

The earliest descriptions of cardiac architecture were biased by the presumption that the organization of ventricular myocardium and skeletal muscle are similar and by the use of relatively crude blunt dissection techniques that reinforced that view. Thus, in the first half of the 20th century, the ventricles were seen to consist of discrete muscle bundles that inserted into the atrioventricular valve ring and followed a complex helicoidal pathway through the ventricular wall $[3,4]$. More recently, the ventricular walls have been incorrectly described as being composed of strap-like muscles [5].

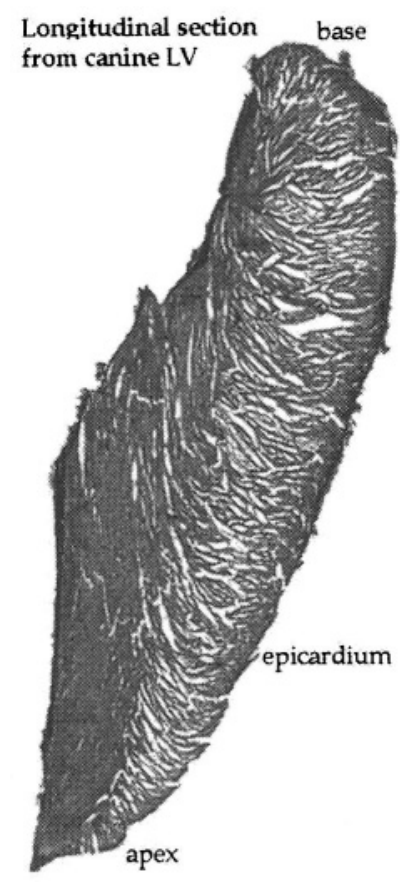

Figure 1. Longitudinal (apex-base) section from the LV of a dog. Section is $100 \mu \mathrm{m}$ thick. Note the radial spaces or cleavage planes between myocardial laminae.

A very different conception emerged from work reported by Lev [6] and Streeter [7]. Ventricular myocardium was described by these workers as a continuum in which myocyte orientation varied smoothly across the ventricular wall from subepicardium to subendocardium. Detailed measurements of the transmural variation of myocyte orientation have been made at limited numbers 
of representative ventricular sites in pig [7], dog [8, 9], primate [10] and human $[11,12]$ hearts. These studies reveal that fiber angle varies by up to $180^{\circ}$ transmurally.

It has been widely assumed that ventricular myocardium is transversely isotropic with respect to the myofiber axis, reflecting the view that myocardium is a continuum in which neighbouring myocytes are uniformly coupled. However, macroscopic observations made over a number of years indicate that there is substantial discontinuity in the muscular architecture of the heart. Sections cut from the ventricles of a range of mammalian species have revealed extensive extracellular gaps that are consistent with an ordered arrangement of muscle layers [4, 13-21]. A typical example is given in Figure 1. This thick apex-base transmural section cut from the left ventricular (LV) free wall of a dog heart reveals an array of discrete tissue layers which run from endocardium to epicardium in an approximately radial direction. While there is extensive branching of muscle layers throughout the section, the radial extent of the gaps between adjacent layers is striking - up to 4 or $5 \mathrm{~mm}$ in the midwall. There have also been reports of large connective tissue septae in the ventricles of a number of species including man [18, 22-24].

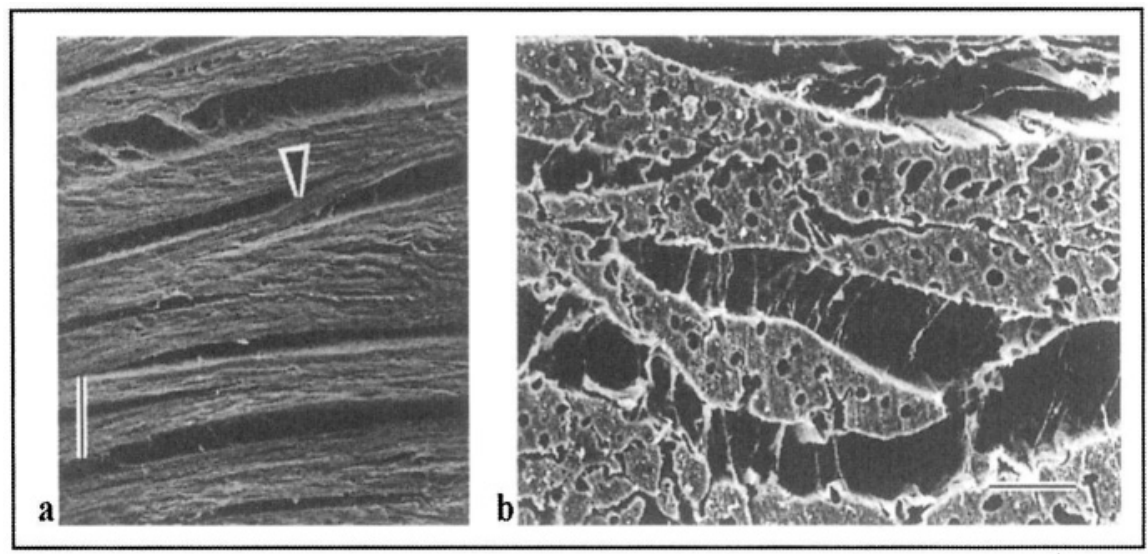

Figure 2. a) SEM of ventricular myocardium sectioned parallel to epicardial surface. Note the laminar arrangement of myocytes. Arrow shows muscle bridge between sheets. Scale bar $100 \mu \mathrm{m}$. b) SEM of ventricular myocardium sectioned transverse to myocyte axis. Capillaries and permysial collagen network are evident (Scale bar $25 \mu \mathrm{m}$ ). (From [18], with permission from the American Physiological Society).

Low-power scanning electron microscopy (SEM) has also been used to image ventricular specimens [18]. As shown in Figure 2, myocytes are arranged in discrete layers, separated by cleavage planes and coupled via an extensive extracellular connective tissue network. There is also direct branching between 
layers with muscle bridges one to two cells thick.

\section{The Cardiac Connective Tissue Hierarchy}

Studies of the extracellular connective tissue matrix in the heart reveal a complex hierarchical organization that reflects the arrangement of cardiac myocytes and the coronary circulation [23, 25-28]. Caulfield and Borg [25] used SEM to reveal the basic organization of this connective tissue network. They described the following three classes of connective tissue organization: (1) interconnections between myocytes, (2) connections between myocytes and capillaries, and (3) a collagen weave surrounding groups of myocytes. Caulfield and Borg [25] noted that myocytes were connected to adjacent myocytes by numerous bundles of collagen 120 to $150 \mathrm{~nm}$ in diameter. These collagen cords were quite straight, with a relatively uniform circumferential distribution and they inserted into the basement membrane of the myocyte close to the Z-line of the sarcomere. Radial collagen cords of similar dimension and appearance also connected myocytes and capillaries. Finally, Caulfield and Borg [25] observed a meshwork of collagen bundles that surrounded groups of three or more myocytes. This external weave was complex, with enclosed myocytes connected to the encompassing network by numerous short, straight, collagen cords. However, Caulfield and Borg [25] observed that adjacent complexes were only loosely coupled by sparse and relatively long collagen fibers.

Robinson and his coworkers [26] went on further to categorize the components of this extracellular connective tissue matrix in an elegant series of studies using light microscopy, together with transmission and scanning electron microscopy. They classified the hierarchy of cardiac connective tissue organization as "endomysium", "perimysium", and "epimysium", using terminology more commonly associated with skeletal muscle.

Endomysium. The cardiac endomysium was seen to incorporate the system of radial collagen cords described by Caulfield and Borg [25], together with a pericellular network of fibers that encompass the myocyte and a lattice of collagen fibrils and microthreads. Robinson and colleagues [26] observed that the radial cords consist of helically wound collagen fibrils that divide close to the cell surface and ramify as part of the pericellular network. In addition to this fine pericellular weave of connective tissue, distinct pericellular cuffs were also seen using light microscopy [26, 27]. It was argued that components of the radial collagen cords also insert into the cell membrane and are tethered to the contractile apparatus at the Z-line [27].

Epimysium. The epimysium was defined as "the sheath of connective tissue that surrounds entire muscles, for example, papillary muscle and trabeculae" [29], and would include components of endocardium and epicardium. It was observed that the epicardium and much of the endocardium consists of a 
thin layer of endothelial cells overlaying a network of randomly oriented collagen fibrils and elastin fibers.

Perimysium. The term perimysium was used to describe the extensive meshwork of connective tissue that surrounds groups of cells and the connections between contiguous cell bundles. Robinson and colleagues [26] observed large coiled bundles of collagen fibers oriented parallel to the long axis of the myocytes in LV myocardium and papillary muscle. These were associated with groups of cells and were defined as "coiled perimysial fibers". A more detailed study of the organization of the coiled perimysial fibers in rat papillary muscle was later reported [28]. A branched network of coiled perimysial fibers 1 to $10 \mu \mathrm{m}$ in diameter was seen to diverge from the muscle-tendon interface and a regular array of radial cords interconnected these fibers with adjacent myocytes and with the endocardial membrane surrounding the papillary muscle. In more recent work, MacKenna and colleagues [30] used a confocal laser scanning microscope to investigate the 3D structure of perimysial collagen fibers from rat LV myocardium, describing their conformation as 'planar waviness rotating around the fiber axis'. Similarly, Hanley and co-workers [31] reconstructed 3D images of rat cardiac trabeculae and demonstrated that the perimysial collagen fibers in those structures were also wavy rather than coiled.

\section{Collagen Types in the Heart}

Collagen is the most abundant component of the extracellular matrix of the heart. There are at least 18 different types of collagen of which five (Types I, III, IV, V and VI) have been identified in myocardium [23, 32]. Whereas types IV and V collagen are components of the basement membrane of cardiac cells, types I and III collagen are the main constituents of the extracellular connective tissue matrix, contributing around 75 to $80 \%$ and 15 to $20 \%$ of total collagen content, respectively [33, 34].

The mechanical and structural properties of type I and type III collagen are quite different. Type I collagen forms thick parallel rod-like fibers 50-150 $\mathrm{nm}$ in diameter and is found in large proportions in tissues requiring high strength such as tendon, bone and skin [32]. Mechanical tests carried out on tendon reveal that type I collagen has a high tensile strength and stiffness. On the other hand, type III collagen is the principal component of the fine collagen fibrils that compose the highly deformable reticular networks characteristic of loose connective tissue and is most abundant in fetal tissues and, in adults, in highly elastic tissues such as skin, blood vessels and lung [32]. Studies with monoclonal antibodies [27, 28, 35-37] indicate that type I and type III collagen are present in both the fine pericellular network of the endomysium and the radial cords that connect myocytes with adjacent myocytes and capillaries. It was demonstrated [27] that type I and type III collagen aggregate in the radial 
collagen cords to form a copolymer. The intensity of antibody staining for type III collagen is stronger for the pericellular network than for the radial collagen cords. On the basis of these results it could be argued that the fine pericellular networks of the cardiac connective tissue hierarchy are predominantly composed of type III collagen.

It has been proposed that the ratio of type I to type III collagen influences mechanical properties [32]. The proportion of type I collagen to the total of type I and type III collagen is least in the fetal heart and increases with age [33]. In bovine skeletal muscle, Light and Champion [38] found that the proportion of type I collagen to total type I and type III collagen is $84 \%$ in epimysium, $72 \%$ in perimysium, and $38 \%$ in endomysium. In nonhuman primates the proportion of type III collagen to total collagen increases in the evolutionary phase of the response to chronic ventricular pressure overload [34]. This is coincident with the proliferation of fine fibrils in the connective tissue weaves of the endomysium and the perimysium.

Of the minor collagen components in the heart, Type IV collagen is found as an open network in basement membranes where it is involved in regulating molecular transport and cell adhesion, while types V and VI are found in the interstitium where they are associated with other collagen types [32].

The rate of collagen turnover in the normal heart is around 5\% per day $[32,39]$. Collagen is synthesized by a number of different types of cell in the heart: Fibroblasts and smooth muscle cells produce all cardiac collagen types, while endothelial cells synthesize all cardiac collagen types except type VI and myocytes produce type IV collagen [32]. Collagen is degraded through both intracellular and extracellular pathways, the latter involving neutral metalloproteinases. Collagen metabolism is highly sensitive to mechanical loading, which can shift the balance between synthesis and degradation, leading to rapid and extensive changes in collagen composition [32]. This is particularly important in the heart remodeling associated with cardiac and cardiovascular diseases such as myocardial infarction, heart failure and hypertension.

\section{Three Dimensional Architecture of Ventricular Myocardium}

In our laboratory, we have developed morphometric techniques intended to provide comprehensive data on the organization of myocytes, blood vessels, and connective tissue in the normal heart and animal models of cardiac disease. The sections below summarize the results obtained from measurements of myocyte and muscle layer orientation.

Myocyte orientation. We have made extensive measurements of ventricular myocyte orientation in a series of normal dog [8] and pig [40] hearts using a technique which explicitly preserves the registration of fiber orientation 
with respect to ventricular geometry. The ventricular walls were progressively shaved away in $0.5 \mathrm{~mm}$ steps and fiber orientations were mapped out at points on the exposed surface, together with the 3D coordinates of those points. For each heart, this resulted in approximately 10,000 data points describing the fiber orientation throughout right and left ventricles. Our results confirm previous findings and demonstrate surprising consistency at corresponding ventricular sites in different dog hearts. However, there is significant local variation of fiber orientation, particularly in the interventricular septum and toward the boundaries of LV and RV free walls and this information cannot be obtained from the restricted data sets published by previous workers.

Our data is obtained by sectioning the ventricular wall parallel to the epicardial tangent plane to reveal the myocardial layers. Fiber orientation is estimated by measuring the orientation of the myocardial layers in that plane on the assumption that the myocyte axis is parallel to the epicardial tangent plane. However, at some sites such as the ventricular base the myocyte axis has a significant transmural component, defined as the imbrication angle [41]. While our heart model allows for the definition of this angle, there have been no extensive measurements of fiber imbrication in the ventricle to date. However, detailed information on myocyte imbrication can been obtained using the 3D reconstruction techniques such as the extended confocal imaging technique outlined below.

Transmural orientation of muscle layers. Muscle layer orientations have also been recorded systematically in thick longitudinal transverse sections from normal dog [18] and pig [40] hearts. A schematic diagram of the layer organization in these transmural sections is given in Figure 3. Muscle layers are generally normal to the ventricular surfaces in the midwall, but the orientation of layers in subendocardial and subepicardial regions varies at different ventricular sites. For instance, muscle layers approach the endocardial surface of the LV cavity with markedly different orientations in the LV free wall and the interventricular septum. Measurements from sections from right and left ventricles reveal a transmural variation of branching between layers with higher branching densities for sub-epicardial and sub-endocardial sections and a minimum near midwall [18].

A further notable characteristic of the laminar organization seen in the ventricles is the regular appearance of small regions in which layers are perpendicular to the predominant direction. While not particularly extensive in canine myocardium, this intersecting pattern is marked in pig myocardium (unpublished observation). The significance of this variation is not yet known though it is likely to be important for accommodating the significant shear deformation which occurs in myocardium throughout the cardiac cycle, since the perpendicular cleavage planes align closely with the two orthogonal planes of maximum shear [42]. 


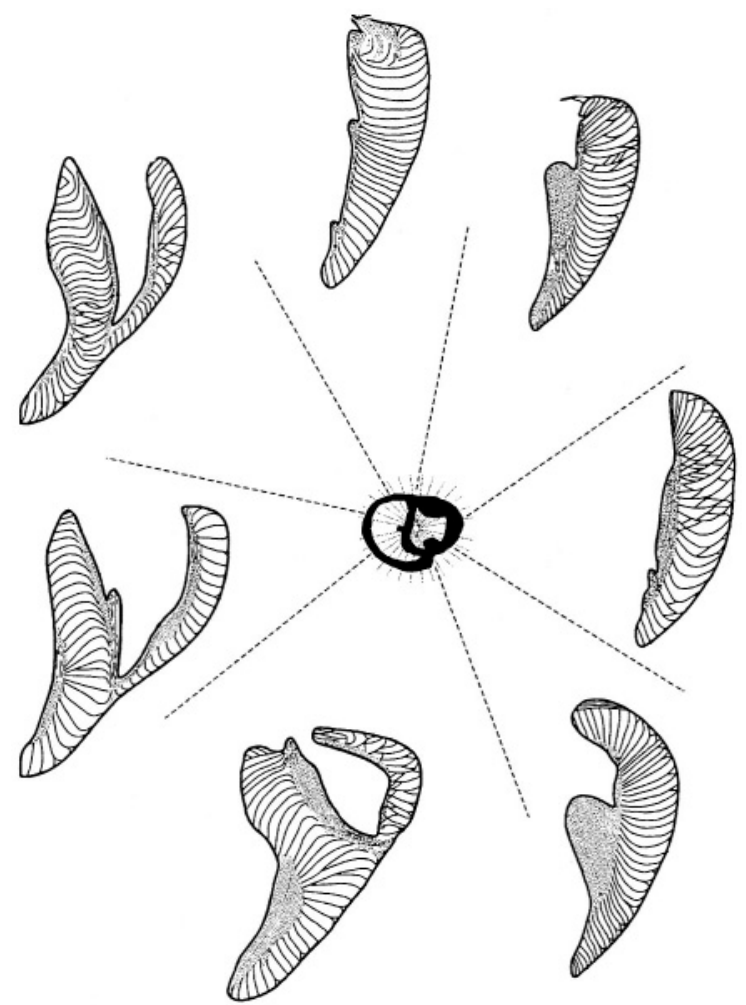

Figure 3. Apex-base transmural sections of the ventricles from a series of circumferential locations in a dog heart. The orientations of myocardial laminae are shown for each section (sketched from original data). (Modified from [18], with permission from the American Physiological Society).

Three dimensional arrangement of muscle layers. Quantitative observations of the 3D organization of muscle layers have been made on segments removed from different LV sites in fixed dog hearts [18]. SEM images for a series of samples from these segments (see Figure 2) reveal consistent patterns in the cellular organization of muscle layers. At this level, the layers appear to be uniform in structure with regular anastomoses between adjacent cells and an ordered interdigitation of cells and capillaries. Layers consist of tightly packed groups of cardiac myocytes aligned so that the cell axis is approximately parallel to the cut edge of the layer and are $4 \pm 2$ myocytes thick at all transmural sites, with branches between layers generally one to two myocytes thick. In those areas where there are appreciable spaces between adjacent layers, their radial extent appears to be considerable and an extensive 
network of connective tissue is observed. Long collagen fibers run between muscle layers and insert into the connective tissue network which surrounds them. These perimysial collagen fibers connect adjacent muscle layers and should not be confused with the thicker coiled perimysial fibers [28] that run approximately parallel to the myocyte fiber axis.

Despite the uniformity of muscle layer organization, the architecture of ventricular myocardium is not homogeneous and shows considerable transmural variation in the density of branching between layers. Specifically, there are twoto three-fold fewer branches in the midwall than in subepicardium (or subendocardium), and the extent of cleavage planes is greatest in the midwall. The branching between adjacent layers is relatively sparse in the midwall and the distance between branches along a layer can be of the order of 2 to $4 \mathrm{~mm}$ in this region. The length of perimysial fibers connecting adjacent muscle layers was also greatest in the midwall. The mean length of perimysial fibers connecting adjacent layers in the subepicardium varied from $13.2 \mu \mathrm{m}$ to $19.8 \mu \mathrm{m}$, but ranged from $22.3 \mu \mathrm{m}$ to $49.4 \mu \mathrm{m}$ in the deep midwall. Moreover the length distribution of these perimysial fibers is considerably wider for the midwall than for the subepicardium.

This work provides more detailed and more systematic information on aspects of myocardial structure and its associated connective tissue hierarchy previously described by Caulfield \& Borg [25] and Robinson and colleagues [26]. It is evident that the layers observed in this work may be equated with the "bundles" of myocytes reported by these workers.

More recently, we have developed a new technique to image extended volumes of myocardium [43]. A confocal microscope is used to acquire contiguous sets of z-series images spanning the upper surface of resin embedded myocardial specimens. This surface is then trimmed and the process is repeated to assemble large, high-resolution image volumes. Digital reslicing, segmentation and volume rendering methods can be applied to the resulting volumes to provide quantitative structural information about the 3D organization of myocytes, extracellular collagen matrix and blood vessel network of the heart which has not previously been available.

Figure 4 is a $3 \mathrm{D}$ reconstruction of a transmural segment $(800 \mu \mathrm{m} x$ $800 \mu \mathrm{m} \times 4.5 \mathrm{~mm}$ ) cut from the LV free wall of a rat heart perfused with picrosirius red [44]. This image, which consists of 650 million voxels at a spatial resolution of $1.5 \mu \mathrm{m}$, provides a striking view of the laminar architecture of the myocardium. The 3D organization of layers varies through the wall to accommodate the transmural variation of fiber orientation. Muscle branches between layers seem to be most dense in subepicardial and subendocardial regions of the specimen, but are relatively sparse in the midwall as reflected by the extent of the cleavage planes between adjacent, midwall muscle layers. The dye, picrosirius red, binds non-sterically to collagen [44] which appears white 
in Figure 4. The extensive collagen network covering the epicardial surface is evident in this figure, as is the convoluted nature of the endocardial surface. In addition, networks of collagen cords that span cleavage planes to connect adjacent muscle layers are clearly visible in Figure 4. Large collagen cords, which are aligned approximately with the myocyte axis and extend over many cell lengths, are also observed in the cleavage planes. These longitudinal cords have a convoluted rather than a coiled appearance. While these do not fall easily into the classification of Robinson and co-workers, they should be regarded as perimysial since they are associated with more than 2 myocytes along their length [28]. Figure 5 indicates that there is a relatively high density of these cords within muscle layers.

It is possible to segment out and display separate structural components within a myocardial volume (see Figure 6) and this provides a powerful basis for efficient morphometric analysis. For instance, it is a relatively trivial matter to quantify the transmural variation of perimysial collagen once it has been segmented out of the extended volume image as shown in Figure $6 \mathrm{~b}$.

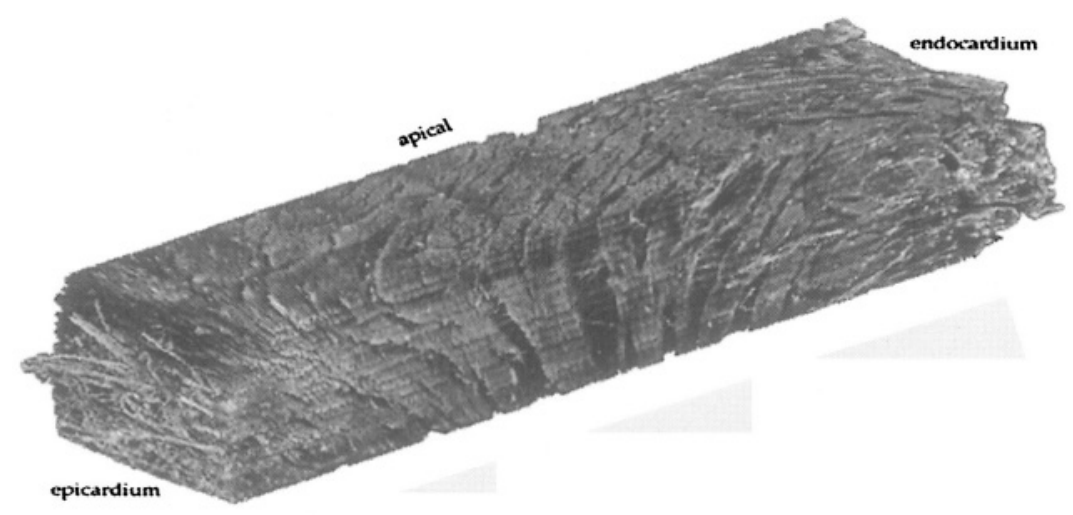

Figure 4. Oblique view of extended volume image from LV of rat heart obtained using confocal microscopy. Note the laminar organization and collagen (white) interconnecting layers of myocytes. The epicardial collagen weave is clearly seen along with cleavage planes between myocardial layers. (From [43], with permission from the Royal Microscopical Society).

Using this approach we have shown that perimysial collagen network is densest in the subendocardium and subepicardium and relatively sparse in the midwall [43]. This also reinforces the critical need to account for sample orientation when carrying out quantitative analysis from 2D microscope images. Because the 3D arrangement of the collagen network varies across the ventricular wall, collagen density should be compared between sections only where the principal orientation of collagen bundles are similar [43]. 


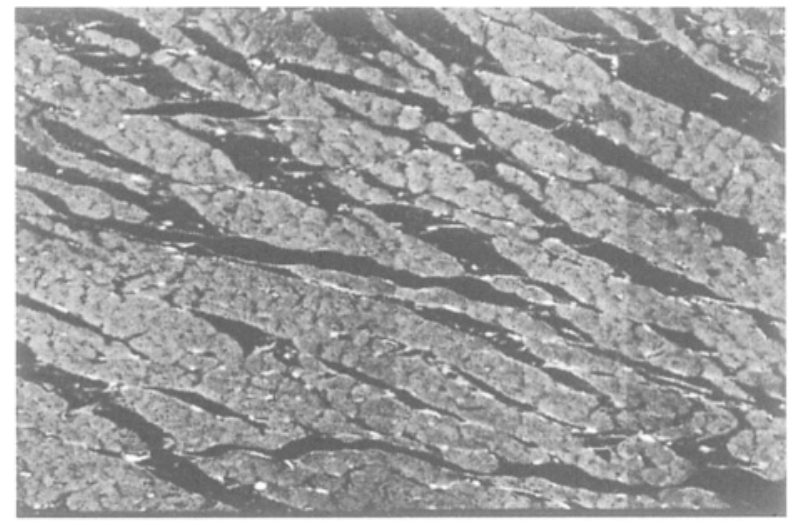

Figure 5. Image slice from LV midwall of rat heart $(800 \times 800 \mu \mathrm{m})$ illustrating laminar organization of myocytes. Plane of optical section is perpendicular to myocyte axis. White dots are perimysial collagen cords running parallel to myocyte axis.
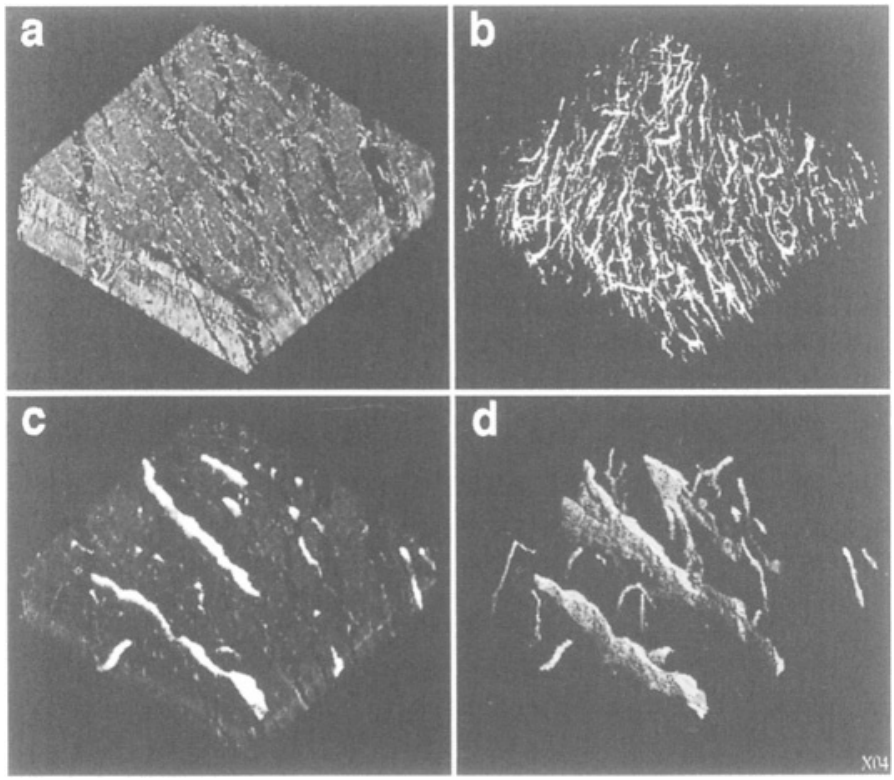

Figure 6. Reconstructed subvolumes $(800 \times 800 \times 100 \mu \mathrm{m})$. In the upper panel collagen is segmented and rendered (a) with and (b) without background due to myocytes. In the lower panel venous sinuses are segmented and rendered, (c) with and (d) without background. (From [43], with permission from the Royal Microscopical Society). 
On the basis of the observations summarized in this chapter, we have developed the conceptual model of myocardial architecture shown in Figure 7. Groups of tightly coupled myocytes are organized into layers with a characteristic transmural direction. These layers twist to accommodate the variation of muscle fiber orientation across the ventricular wall and are

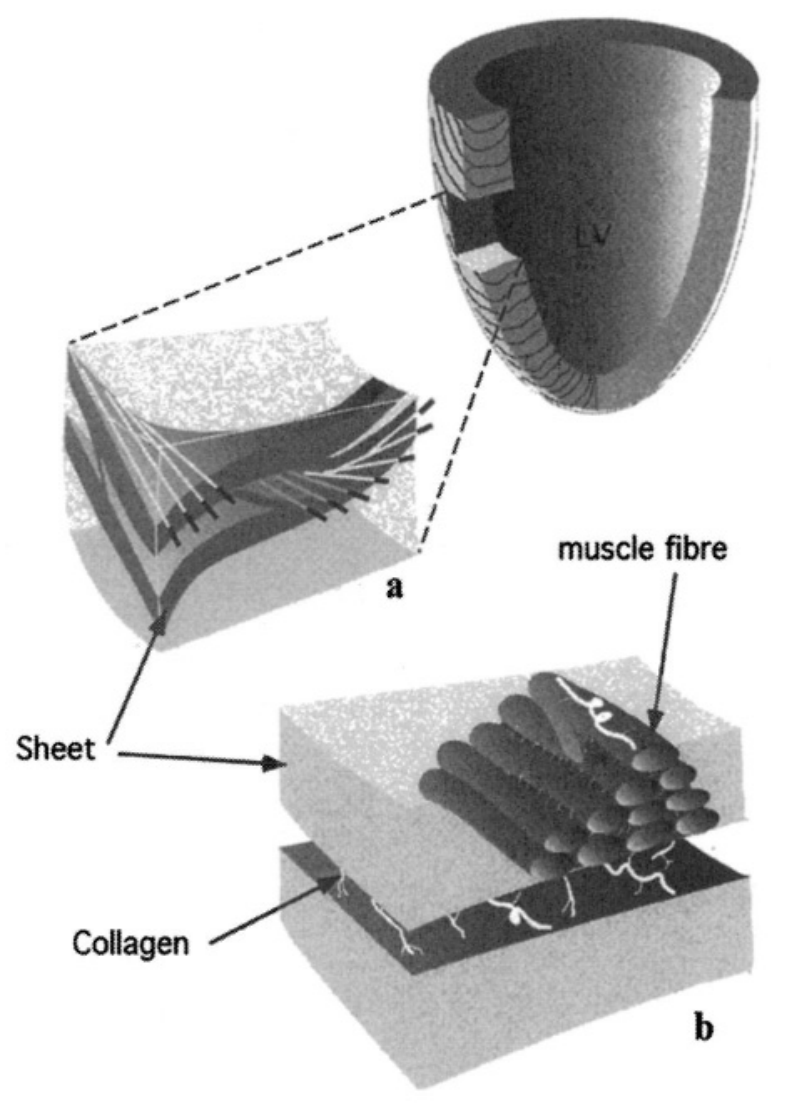

Figure 7. Schematic of cardiac microstructure. (a) A transmural block cut from the ventricular wall shows orientation of fibers. Note the transmural variation in fiber angle. (b) The muscle fibers are shown forming a layer three to four cells thick. Endomysial collagen is shown connecting adjacent cells within a sheet while perimysial collagen links adjacent sheets. (Modified from [18]).

interconnected via relatively sparse muscle bridges. The extracellular connective tissue network of the heart reflects the hierarchy of myocyte architecture and plays a major role in organizing this structure. Endomysial collagen registers the myocytes within a layer. A network of perimysial collagen cords tether adjacent 
muscle layers and a further population of convoluted perimysial cords is oriented in the direction of the myocyte axis. It should not be imagined that the $3 \mathrm{D}$ organization of muscle layers illustrated in this figure is the only possible arrangement: within such a hierarchy of muscle layers a range of various transmural connections is possible.

\section{Myocardial Architecture and Cardiac Function}

There is a considerable body of indirect experimental evidence that suggests that the characteristics and extent of the extracellular connective tissue matrix are important determinants of diastolic and systolic ventricular function. Robinson and his coworkers [26-29,45] proposed specific mechanical roles for the cardiac connective tissue matrix based on their anatomical observations. The collagen network was seen as a "strain-locking" system, which resists the extension of myocytes beyond sarcomere lengths of $2.25 \mu \mathrm{m}$ while allowing

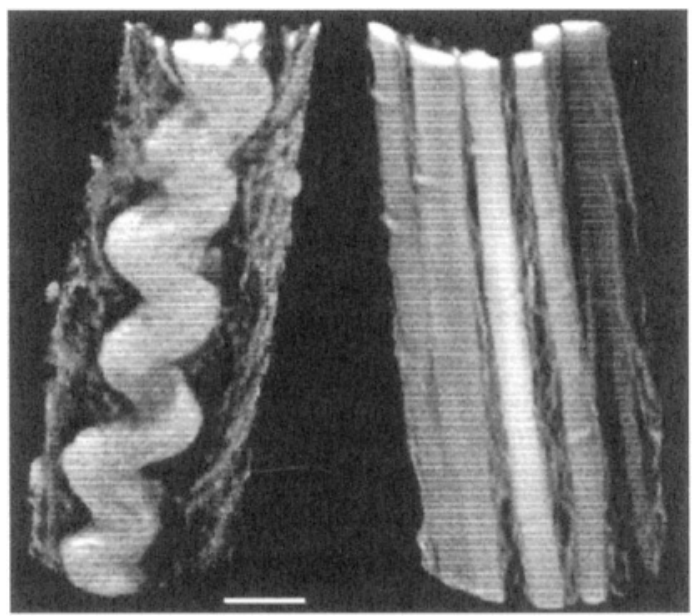

Figure 8. 3D reconstruction of typical permysial collagen fibers from rat ventricular trabeculae fixed at near-resting sarcomere length (left) and at an extended sarcomere length (right). (Scale bar $10 \mu \mathrm{m}$.) (From [31], with permission from the Physiological Society).

relatively free extension up to this length. Thus, when myocardium is stretched, the biaxially organized connective tissue networks of the epimysium, perimysium, and endomysium are rearranged to align more closely with the muscle-fiber direction (cargo net hypothesis). In addition, coiled perimysial fibers in ventricular myocardium and papillary muscle, which are obliquely or longitudinally aligned with respect to the long axis of muscle fibers, become less 
convoluted. Robinson and colleagues $[26,28]$ noted that the end-points of both these changes in connective tissue configuration are correlated with a striking increase in the axial stiffness of papillary muscles when extension exceeds $15 \%$ of slack length. MacKenna and colleagues [30] used 2D light microscopy techniques to demonstrate that, in the rat LV, convoluted perimysial collagen cords aligned with the myocyte axis straighten as sarcomere length approaches $2.25 \mu \mathrm{m}$. This mechanism is clearly illustrated in Figure 8 [31]. In this case, Hanley and colleagues used confocal microscopy to reconstruct rat ventricular trabeculae in 3D for different axial extensions.

It is most probable that each of the components of the connective tissue hierarchy contributes to the mechanical properties of passive myocardium. Robinson and colleagues argued that the epimysium protects sarcomeres from being overstretched [26]. Caulfield and Borg [25] argued that the short radial collagen cords of the endomysium ensure that adjacent myocytes remain in registration providing a mechanism for uniform distribution of strain at the cellular level. They saw the perimysial network as organizing individual myocytes into a functional unit and providing a framework for distributing the contractile force within the ventricular wall. It is also argued [45] that the extracellular connective tissue matrix stores energy during systole and contributes to the elastic recoil of the ventricles during rapid filling. One of the lines of argument involves a straightforward corollary of the cargo net hypothesis. Since the volume occupied by the contractile lattice is relatively constant, the cross-sectional area of the myocyte increases when shortening occurs. It was argued that this dimensional change will lead to a realignment of the pericellular network of the endomysium with collagen fibrils now preferentially oriented transverse to the muscle-fiber direction. (A similar reorganization was also seen to apply for the perimysial network surrounding groups of muscle cells.) There was further speculation that the systolic deformation of the ventricle could impose tensile load on the collagen cords that tether adjacent myocytes [45] and compress the coiled fibers and tendons of the perimysium [28]. It was postulated that the potential energy storage associated with any of these mechanisms could oppose further shortening and tend to restore the myocardium to its resting state.

It would seem obvious that the major component of passive myocardial stiffness resides within the extracellular connective tissue matrix. However, it is now evident that intracellular structures also contribute significantly to myocardial stiffness. Of particular interest is the huge protein titin which has been viewed as an adjustable spring which actively modulates aspects of myocardial stiffness [46]. The intercellular and extracellular domains should not, of course, be seen as independent: they are coupled both in parallel and in series through transmembrane proteins such as integrins which connect the extracellular matrix to the cytoskeleton. These molecules transmit force between 
the domains, but also play a role as mechano-receptors, transducing force information and activating cellular signal pathways [2].

Recent bioengineering analyses provide overwhelming evidence that the effectiveness of cardiac mechanical function is intimately associated with the 3D arrangement of myocytes in the heart. The ventricles experience substantial dimensional change throughout the cardiac cycle and because they are thickwalled structures, there are significant transmural gradients of strain. In the LV, for example, end-diastolic circumferential extension can be more than three times greater at the endocardium than the epicardium and, in general, is significantly less than longitudinal extension at all transmural sites. It has been argued that the changing orientation of myoctyes across the ventricular wall and the associated torsion which occurs as the heart contracts act to normalize sarcomere length changes throughout the cardiac cycle and hence normalize myocyte stress and work across the ventricular wall [47, 48]. It is also argued that transmural coupling through the connective tissue matrix is necessary for contraction of the spirally oriented myocytes to be translated into effective pumping action [49]. Experimental work has shown that when strains are calculated with respect to a local structurally-oriented coordinate system that sarcomere strains are indeed uniform across the ventricular wall during diastole [50] and systole [51-53].

The laminar organization of ventricular myocardium also appears to play a critical role in accommodating shear deformations in the heart wall. This hypothesis accompanied a number of early observations of laminar structure in the heart [13, 14, 17]. Caulfield and Borg also stated that the loose collagen connections between groups of myocytes facilitate the large shearing deformations that are necessary for normal diastolic and systolic ventricular function [25]. More specifically, it has been argued the substantial changes in ventricular wall thickness that occur during the cardiac cycle are possible only because of slippage and rearrangement of adjacent muscle layers in the subendocardial regions of the ventricles. Spotnitz and colleagues investigated this idea and demonstrated that the transmural orientation of cleavage planes between muscle layers became progressively more oblique as the wall thinned during diastole [20]. Three-dimensional strain analysis reveals substantial shearing between adjacent subendocardial layers in systole [51, 52, 54] and diastole [52]. The wall slippage mechanism has also been invoked to explain aspects of the regional ventricular dilatation and local wall thinning which follow myocardial infarction $[17,19,21]$. The shear properties of passive LV myocardium have been related to its laminar structure using midwall specimens in which the principal features of myocardial architecture were preserved [55]. This study indicates that passive myocardium has nonlinear, viscoelastic, anisotropic shear properties with least resistance to simple shear displacements imposed in the plane of the myocardial layers. Analyses of the data suggests that 
simple shear deformation is resisted by elastic elements aligned with the microstructural axes of the tissue, and that the inherent shear stiffness of passive myocardium is relatively small.

The laminar organization of ventricular myocardium is believed also to affect the propagation of electrical activation through the heart and this point has been raised in an associated context by Spach and co-workers $[24,56]$. If it is assumed that muscle layers are electrically insulated by the connective tissue surrounding the layers, depolarization can spread to adjacent laminae only via direct muscle branches. Therefore, the path required to traverse a given distance perpendicular to the layers will be convoluted, particularly in the mid-ventricular wall where the frequency of branching is relatively low. This would not influence normal cardiac electrical activation since the endocardial surface of the ventricles are rapidly excited via the Purkinje fiber network and the radial arrangement of muscle layers will ensure an efficient transmural spread of activation. However, the structural anisotropy of ventricular tissue may well contribute to the patterns of electrical activation associated with various arrhythmias. Computer modeling studies in which the effect of the laminar structure was investigated indicate that this is the case [57]. Furthermore, this study also indicated that activation immediately after, and in the region of, an ectopic stimulus proceeds in a non-uniform manner and generates fractionated extracellular electrograms because of the discontinuities introduced by the laminar structure. This study also provided an exciting new insight into the mechanism of action of a defibrillating shock. When such a shock is applied across the heart wall, depolarizing membrane potential steps occur at each boundary between myocardial laminae, and subsequently depolarizing the bulk of the myocardium within a few milliseconds of the shock, a necessary condition for effective defibrillation.

In conclusion, the architecture of the heart has a profound effect on its mechanical and electrical function. The cardiac extracellular matrix plays a central role in the maintenance and adaptation of this structure in the normal heart and is intimately linked with the remodeling and associated impairment of cardiac function that commonly accompanies diseases such as heart failure and hypertension. In order to understand these processes more fully we need conceptual and experimental models that will enable us to integrate the complex interactions between structure and function to which the cardiac extracellular matrix contributes at the whole-heart level. Before this can be done, however, more detailed and more quantitative information about the cellular and molecular processes that underlie the biology of the cardiac extracellular matrix is required.

\section{References}

1. Costa, K.D., J. Holmes, and A.D. McCulloch, Modeling cardiac mechanical properties 